\title{
The Inseparable Bond Between Research and Medical Education
}

Mindy George-Weinstein, PhD

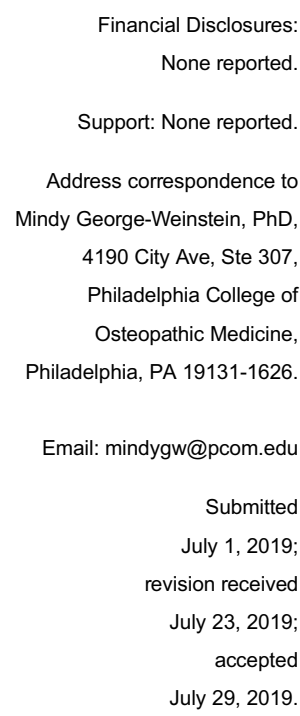

July 29,2019

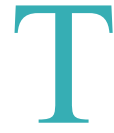
his issue of The Journal of the American Osteopathic Association's Engage Initiative $^{1}$ features the multidisciplinary research program of the Philadelphia College of Osteopathic Medicine (PCOM), with contributions from the fields of osteopathic manipulative medicine, neuropathology, and education, and an analysis of the effect of research on residency matching. Philadelphia College of Osteopathic Medicine has a long-standing commitment to the discovery process that leads to innovations in health care through basic, translational, clinical, behavioral, education, and community-based research. An essential component of faculty research at PCOM is mentoring students in study design and execution, information retrieval and appraisal, and dissemination of information.

The PCOM Engage Initiative issue featuring PCOM research includes 2 articles describing structure and function relationships. Noto-Bell et $\mathrm{al}^{2}$ studied the effects of osteopathic manipulative treatment (OMT) via muscle energy technique (MET) on ankle range of motion and flutter kick performance in pediatric competitive swimmers. MET, using the principle of post-isometric relaxation, was selected as the treatment of choice because it could be taught to and applied by coaches and trainers. Treatment significantly increased ankle plantarflexion in swimmers with restricted range of motion. The authors suggest that a longer recovery time after MET may reduce muscle fatigue and improve performance.

Selby et $\mathrm{al}^{3}$ discuss the effects of OMT on lower back pain in pregnant women and athletes with vertebral displacement in the context of evolutionary variations in lumbar spine morphology among the superfamily of Hominoidea (apes). The authors emphasize that modern humans have unique adaptations in the lumbar spine, including lordosis, that permit bipedality but also lead to injuries not seen in apes. Lordosis may be exaggerated with clinical consequences in obese and pregnant individuals or reduced after surgical treatment for scoliosis. The authors review studies demonstrating that OMT reduces lower back pain in athletes and women in the third trimester of pregnancy, and recommend MET and counterstrain techniques for presurgical management of patients with flatback syndrome.

Moving from the macroscopic to microscopic, Balin et $\mathrm{al}^{4}$ contribute a case report of mixed neuropathology in a deceased patient that had been diagnosed with Lewy body disease based on clinical presentation. ${ }^{4}$ Histological analyses revealed cytoplasmic inclusions found in Lewy body and Alzheimer's diseases, as well as other neurodegenerative processes. ${ }^{4}$ Chlamydia pneumoniae also was detected in this brain. Finding bacteria in the brain of an individual with Lewy body disease is consistent with previously demonstrated correlations between infection and lateonset dementia of the Alzheimer type. ${ }^{5}$

The issue also contains 3 papers focusing on the education of health professionals. Methods of pedagogy are constantly evolving, and each innovation must be accompanied by measurements of its effectiveness. Butts et $\mathrm{al}^{8}$ present outcomes data of their transition course, "Introduction to Clinical Clerkship." Students were enrolled in the course after completing the second year of medical school and before beginning clinical rotations The course integrated basic science concepts with clinical medicine and provided students with a basic understanding of rotation responsibilities. Students reported increased confidence and ease of transition into the clinical setting and were perceived by precepting faculty as better prepared to perform their clinical responsibilities.

Felgoise et $\mathrm{al}^{6}$ describe outcomes of an interprofessional education (IPE) course developed and implemented by a multidisciplinary group of 
PCOM faculty. IPE is necessary for training students to work in teams, meet competency standards, and improve patient assessment and outcomes. ${ }^{7}$ Students from the doctor of osteopathic medicine, clinical psychology, mental health counseling, and physician assistant studies programs attended sessions on a variety of health care topics and participated in collaborative, small-group activities. Surveys administered at the end of the course revealed an appreciation for the perspectives of other healthcare professionals.

The third education related paper by Matthews et $\mathrm{al}^{9}$ describes a model for integrating instruction in the principles and practice of research into undergraduate medical education. While a scholarly approach to clinical practice is broadly recognized as essential for keeping pace with and applying innovations in health care delivery, mechanisms for imparting research-related knowledge vary widely between medical schools. The paper begins with a narrative review of the requirements for research and scholarly activity in osteopathic undergraduate medical education. The authors propose a curricular model in which guided, independent learning of foundational, research-related topics is followed by team-based activities and a smaller group assignment of designing a study. ${ }^{9}$ The goals of the proposed model are to encourage students to participate in research, prepare them for the requirement of scholarly activity as residents, and promote the practice of evidence-based medicine.

Participation in research may also enhance osteopathic medical students' competitiveness for residency positions. Matthews et $\mathrm{al}^{10}$ performed an analysis of data from the 2016 and 2018 National Resident Match Program Charting Outcomes reports to identify potential relationships between match status, medical degree, mean number of research accomplishments and experiences, and specialty. Research accomplishments correlated with matching to a greater extent for allopathic than osteopathic applicants. With the single accreditation system taking effect in 2020, research training and accomplishments may become more significant factors for osteopathic medical students in the residency application process.

Traditionally, medical students sit in lecture halls and in front of their computers absorbing information that faculty have distilled from multiple sources to include the most up-to-date and clinically relevant material. Immersion in team-based and active learning groups leads to the same end of gaining knowledge; however, students determine what information is relevant, retrieve it themselves, and teach their classmates what they have learned. ${ }^{11}$ An introduction to the application of clinical skills is provided in various formats, including patient simulations and transition courses. These learning environments are essential components of preparing students for clinical rotations and beyond; however, the pressure of assimilating vast amounts of information during preparation for course and board examinations leaves little time for wonder.

Curiosity lies at the heart of discovery. The pathway to discovery begins with questions and predictions, but also requires intellectual discipline that defines and refines the approach to test hypotheses, use appropriate tools to examine data without bias, and interpret findings in a broad and imaginative context. These aspects of the research process should be woven into the fabric of our educational mission, with the goal of preparing students to appreciate and contribute to the generation of new knowledge that leads to disease prevention and improvements in detection, diagnosis, and treatment.

The inseparable bond between research and medical education is recognized by undergraduate and graduate medical education accrediting bodies, faculty, and residency directors. However, integrating instruction in the principles and practice of research into an already dense curriculum presents significant challenges. A fundamental shift in the way we present information, with inclusion of questions that reach beyond our current knowledge to inspire curiosity and critical thinking, will contribute to the development of osteopathic medical students as scholars that strive for best practices and contribute to innovations in health care delivery. (doi:10.7556/jaoa.2019.108) 


\section{References}

1. Orenstein R. ENGAGE initiative: showcasing osteopathic scholarly activity. J Am Osteopath Assoc. 2016;116(5):276-277. doi:10.7556/ jaoa.2016.054

2. Noto-Bell L, Vogel BN, Senn DE. effects of post-isometric relaxation on ankle plantarflexion and timed flutter kick in pediatric competitive swimmers. J Am Osteopath Assoc. 2019;119(9):569-577. doi:10.7556/ jaoa.2019.100

3. Selby MS, Gillette A, Raval Y, Taufiq M, Sampson MJ. The modern medical consequences of the ancient evolution of a long, flexible lumbar spine. J Am Osteopath Assoc. 2019;119(9):622-630. doi:10.7556/jaoa.2019.105

4. Balin BJ, Hammond CJ, Galluzzi, KE. Intriguing mixed neuropathology in a case of Lewy body disease. J Am Osteopath Assoc. 2019;119 (9):632-636. doi:10.7556/jaoa.2019.106

5. Balin BJ, Hammond CJ, Little CS, et al. Chlamydia pneumoniae: an etiologic agent for late-onset dementia. Front Aging Neurosci. 2018;10:302. doi:10.3389/fnagi.2018.00302

6. Butts CA, Brady JJ, Speer JJ, et al. Introduction to clerkship: bridging the gap between preclinical and clinical medical education. J Am Osteopath Assoc. 2019;119(9):578-587. doi:10.7556/jaoa.2019.101
7. Felgoise SH, Branch J, Poole A, Levy L, Becker, M. Practicing interprofessional education: collaboration and learning in action. J Am Osteopath Assoc. 2019;119(9):612-619. doi:10.7556/ jaoa.2019.106

8. Schmitt M, Blue A, Aschenbrener CA, Viggiano TR. Core competencies for interprofessional collaborative practice: Reforming health care by transforming health professionals' education. Academic medicine: J Assoc Am Medical Colleges. 2011;86(11):1351.

9. Matthews CN, Estrada DC, George-Weinstein M, Claeson K. Integrating the principles and practice of research into undergraduate medical education: a narrative review and proposed model for implementation. J Am Osteopath Assoc. 2019;119(9):598-611. doi:10.7556/jaoa.2019.103

10. Matthews CN, Estrada DC, George-Weinstein M, Claeson KM, Roberts $M B$. Evaluating the influence of research on match success for osteopathic and allopathic applicants to residency programs. J Am Osteopath Assoc. 2019; 119(9):588-596. doi:10.7556/jaoa.2019.102

11. Gifford KA, Fall LH. Doctor coach: a deliberate practice approach to teaching and learning clinical skills. Acad Med. 2014;89(2):272-276. doi:10.1097/ACM.0000000000000097.

() 2019 American Osteopathic Association

\section{Peer Reviewers Wanted}

Peer reviewers are physicians, basic scientists, and other healthcare professionals who critically evaluate the scientific quality and clinical significance of research submitted to The Journal of the American Osteopathic Association for publication. The JAOA is currently looking for such individuals who are interested in volunteering as peer reviewers. For additional information, visit http://jaoa.org/ss/reviewers.aspx or e-mail jaoa@osteopathic.org for more information. 